\title{
DUAL MINIMAL CURVES
}

\author{
Ali Çalışkan \\ Deparrment of Mathematics, Ege University, 35100 Bornova, Izmir, Turkey \\ caliskan@sci.ege.edu.tr
}

\begin{abstract}
In this study, a dual minimal curves of a dual unit sphere, and the minimal ruled surfaces corresponding it are defined, and represented that dual unit tangent, binormal and surface normal vectors, related to these, are dual isotropic lines. E.Study theorem is also given, for dual minimal curves, belonging to minimal curves and we give general solution of differential equation of this curves.
\end{abstract}

Keywords- Dual minimal curves, dual unit sphere, minimal ruled surfaces.

\section{TANGENT AND BINORMAL OF A DUAL CURVE}

Let's take a line $\vec{X}$, determined in a moving space $H$, and $H^{\prime}$ be a fixed space. The line $\vec{X}$ on $H^{\prime}$ draws a ruled surface, called orbit surface

$$
(X): \quad \vec{X}=\vec{X}(t)=\vec{X}(t)+\varepsilon \vec{X}^{*}(t)=\left(X_{1}(t), X_{2}(t), X_{3}(t)\right)
$$

on one parameter motion $H / H^{\prime}$ of $H$ with respect to $H^{\prime}$. Let's take moving and dual fixed unit spheres $K$ and $K^{\prime}$, and a point $X$ of $K$, corresponding $H$ and $H^{\prime}$, and line $\vec{X}$ of $H$ respectively. Then, the point $X$ on $K^{\prime}$ draws dual curve $(X)$, given (1), on one parameter motion $K / K^{\prime}$ of $K$ with respect to $K^{\prime}$, where $t \in R,[1,2,3]$.

A changing of line $\vec{X}$ (or $X$ point) with respect to $H^{\prime}$ (or $K^{\prime}$ ), that is, the tangent of dual curve $(X)$ at the point $X$ is given by

$$
\frac{d_{f} \vec{X}}{d t}=\vec{\Psi} \wedge \vec{X}=\vec{X}_{f}
$$

where $\vec{\Psi}$ is dual pfaffian vector. The dual unit tangent and the dual unit binormal of dual curve $(X)$ at the same point are

$$
T=\frac{\dot{\vec{X}}_{f}}{\|\dot{\vec{X}}\|} \quad \text { and } \quad B=\frac{\dot{\vec{X}}_{f} \wedge \ddot{\vec{X}}_{f}}{\left\|\dot{\vec{X}}_{f} \wedge \ddot{\vec{X}}_{f}\right\|}
$$

[4].

\section{ISOTROPIC OR DUAL MINIMAL CURVES (SURFACES)}

In this section, some definitions and properties(characteristics) in the reel spaces which are related with our topic $[5,6,7,8,9]$, have generalized to the dual spaces.

\section{Definition 2.1}

If the dual unit vector $\vec{X}=\vec{X}(t)$ is a differentiable function of imaginary variable, given by

$$
t=t_{1}+i t_{2}
$$

we will call it, which is generated by dual complex vectoral function $\vec{X}(t)$, as a dual complex ruled surface or a dual imaginary curve of dual unit sphere $K^{\prime}$. 


\section{Definition 2.2}

We call isotropic or dual minimal curve, on the dual unit sphere $K^{\prime}$, which the square distance between any two point of it, is equal to zero, and also a ruled surface corresponding this curve called isotropic or minimal ruled surface. only if

A dual curve (ruled surface) can be a dual isotropic curve (ruled surface), if and

$$
d S^{2}=d X_{1}^{2}+d X_{2}^{2}+d X_{3}^{2}=0
$$

is satisfied.

\section{DUAL SPHERICAL MINIMAL CURVES}

The parameter equation of a dual sphere (line congruence) can be taken as

$$
\vec{X}(u, v)=\frac{\left[X_{1}=(u v-1), X_{2}=(v+u), X_{3}=i(u v+1)\right]}{v-u}
$$

where $u, v \in R$, then if we differentiate (6), we have

$$
d \vec{X}=\frac{1}{(v-u)^{2}}\left\{\begin{array}{l}
d X_{1}=\left[\left(v^{2}-1\right) d u+\left(1-u^{2}\right) d v\right] \\
d X_{2}=2(v d u-u d v) \\
d X_{3}=i\left[\left(1+v^{2}\right) d u-\left(1+u^{2}\right) d v\right]
\end{array}\right.
$$

and the square of dual spherical arc length can be given as

$$
d S^{2}=\frac{1}{(v-u)^{2}}\left[\sum_{i=1}^{3} d X_{i}^{2}\right]=\frac{4}{(v-u)^{2}} d u d v
$$

Hence we have following result.

\section{Result 3.1}

The minimal curves of the dual unit sphere are the dual isotropic lines of this sphere, that is, these are $u=$ const. and $v=$ const. coordinate lines of it. In the other words, the minimal ruled surfaces of line congruence corresponding this sphere are the isotropic or minimal ruled surfaces of the line congruence, that is, these are $u=c o n s t$. and $v=$ const. coordinate ruled surfaces of it.

\section{Theorem 3.1}

The dual tangent (differential) vectors at the dual smooth point of a dual minimal curve $(X)$ are isotropic lines. Or the normal lines to surface at the striction point of the smooth line of minimal ruled surface $[X]$ is isotropic lines.

Proof Let

$$
\vec{X}(t)=\sum_{i=1}^{3} X_{i}(t) E_{i}
$$

be one dual minimal curve (ruled surface), where $t \in C$ and $\vec{X} \in D^{3}$.

Because of (5), we have

$$
d S^{2}=d \vec{X}^{2}=0 \text {. }
$$

Since it is $\vec{X}^{\prime}=\frac{d \vec{X}}{d t} \neq 0$ at the smooth point (on line), we get 


$$
\vec{X}^{\prime 2}=0
$$

Hence, we reach the following definition.

\section{Definition 3.3}

It is called minimal ruled surfaces that normalies to the surface at the striction point of smooth straight lines of it, are consist of isotropic straight lines. And It is called minimal curves ,corresponding these ruled surface , on the sphere, of which tangents at the smooth points are consist of isotropic straight lines.

\section{Theorem 3.2}

Each of dual unit binormal vectors of a dual curves $(X)$ is isotropic straight line and it is perpendicular to itself.

Proof Taking derivative of (9), with respect to $t$, is

$$
\left\langle\vec{X}^{\prime}(t), \vec{X}^{\prime \prime}(t)\right\rangle=0 \text {. }
$$

Because of, the dual osculating plane at the smooth point of dual minimal curve $(X)$ is parallel both dual vectors $\vec{X}^{\prime}(t)$ and $\vec{X}^{\prime \prime}$, since (10), the dual vector $\vec{X}^{\prime \prime}(t)$, generally $\vec{X}^{\prime \prime}(t) \neq 0$ is satisfying, is perpendicular to $\vec{X}^{\prime}(t)$, that is, $\vec{X}^{\prime \prime}(t)$ is parallel to dual principal normal vector at the smooth point.

On the other hand, the square of the dual vector $\vec{X}^{\prime}(t) \wedge \vec{X}^{\prime \prime}(t)$ which determine the dual binormal vector at the smooth point, is $\left[\vec{X}^{\prime} \wedge \vec{X}^{\prime \prime}\right]^{2}=\vec{X}^{\prime 2} \vec{X}^{\prime \prime 2}-\left\langle\vec{X}^{\prime}, \vec{X}^{\prime \prime}\right\rangle^{2}$, since (9) and (10)

is satisfied.

$$
\left[\vec{X}^{\prime}(t) \wedge \vec{X}^{\prime \prime}(t)\right]^{2}=0
$$

Hence we get the following result.

\section{Result 3.2}

Dual binormal vectors of a dual minimal curve $(X)$ are on the dual osculating plane (consisting of $\vec{X}^{\prime}, \vec{X}^{\prime \prime}$ ). In the other words, this osculating plane which passes through their own normal, is a dual isotropic plane.

Again due to (9) and (10), we get

$$
\vec{X}^{\prime} \wedge\left(\vec{X}^{\prime} \wedge \vec{X}^{\prime \prime}\right)=\left\langle\vec{X}^{\prime}, \vec{X}^{\prime \prime}\right\rangle \vec{X}^{\prime}-\vec{X}^{\prime 2} \vec{X}^{\prime \prime}=0 .
$$

Hence, we reach the following result.

\section{Result 3.3}

If the dual binormal vector which is isotropic straight line of a dual minimal curve, is on the dual osculating plane which is a isotropic plane, this plane has only one isotropic straight line which passes through giving smooth point, and this is determined by the tangent at this smooth point of dual minimal curve. So that, this dual binormal vector is coincide with this tangent. 


\section{Result 3.4 (E-Study Theorem)}

If the dual osculating planes of a dual curve is consist of isotropic planes, this curve is either a isotropic curve or a curve of dual isotropic plane.

Proof If the dual osculating planes of a dual curve are dual isotropic planes; the dual normal vectors $\vec{X}^{\prime} \wedge \vec{X}^{\prime \prime}$ are paralel to dual osculating plane. That is, at any point of dual curve, we get

$$
\left(\vec{X}^{\prime} \wedge \vec{X}^{\prime \prime}\right)^{2}=0 \text {. }
$$

Hence taking derivative from (12) gives

$$
\left\langle\vec{X}^{\prime} \wedge \vec{X}^{\prime \prime}, \vec{X}^{\prime} \wedge \vec{X}^{\prime \prime \prime}\right\rangle=0
$$

On the other hand, since we have the relation

$$
\left(\vec{X}^{\prime} \wedge \vec{X}^{\prime \prime}\right) \wedge\left(\vec{X}^{\prime} \wedge \vec{X}^{\prime \prime \prime}\right)=\left(\vec{X}^{\prime}, \vec{X}^{\prime \prime}, \vec{X}^{\prime \prime \prime}\right) \vec{X}^{\prime} .
$$

If we take the vectoral product of both side of (14) with $\vec{X}^{\prime} \wedge \vec{X}^{\prime \prime}$, since (13) and (12) left side is equal to zero, and it is

$$
\overrightarrow{0}=\left(\vec{X}^{\prime}, \vec{X}^{\prime \prime}, \vec{X}^{\prime \prime \prime}\right)\left[\vec{X}^{\prime} \wedge\left(\vec{X}^{\prime} \wedge \vec{X}^{\prime \prime}\right)\right]=\left(\vec{X}^{\prime}, \vec{X}^{\prime \prime}, \vec{X}^{\prime \prime}\right)\left[\left\langle\vec{X}^{\prime}, \vec{X}^{\prime \prime}\right\rangle \vec{X}^{\prime}-\vec{X}^{\prime 2} \vec{X}^{\prime \prime}\right]
$$

dual vectors $\vec{X}^{\prime}$ and $\vec{X}^{\prime \prime}$ is not parallel to each other. In this case :

Either it is $\left(\vec{X}^{\prime}, \vec{X}^{\prime \prime}, \vec{X}^{\prime \prime \prime}\right)=0$ along the dual curve, and so a dual curve of a dual plane is a point at issue, or it is that $\left\langle\vec{X}^{\prime}, \vec{X}^{\prime \prime}\right\rangle=0$ and $\vec{X}^{\prime 2}=0$ at the every point, that is, a dual minimal curve is obtained.

\section{DETERMINING THE DUAL MINIMAL CURVES ON THE SPACE}

We have defined the dual minimal curve on the perpendicular coordinate system with the relation (5). Now for general solution of this differential equation, we can write (5), as in the following form

$$
\frac{d X_{2}+i d X_{3}}{d X_{1}}=-\frac{d X_{1}}{d X_{2}-i d X_{3}}=t
$$

where $i, t \in C$ and if we take

$$
X_{2}^{\prime}=\frac{d X_{2}}{d X_{1}}, \quad X_{3}^{\prime}=\frac{d X_{3}}{d X_{1}} .
$$

from the relation (15), we have

$$
X_{2}^{\prime}+i X_{3}^{\prime}=t, \quad X_{2}^{\prime}-i X_{3}^{\prime}=-1 / t
$$

Hence

$$
X_{2}^{\prime}=\frac{1}{2} \cdot \frac{t^{2}-1}{t}, \quad X_{3}^{\prime}=\frac{1}{2 i} \cdot \frac{t^{2}+1}{t}
$$

or

$$
\frac{d X_{1}}{2 t}=\frac{d X_{2}}{t^{2}-1}=\frac{i d X_{3}}{t^{2}+1}=G(t) d t
$$

is found. And so we get

$$
X_{1}=2 \int t G(t) d t, \quad X_{2}=\int\left(t^{2}-1\right) G(t) d t, \quad X_{3}=-i \int\left(t^{2}-1\right) G(t) d t .
$$

If we take $G(t)=F^{\prime \prime \prime}(t), t=u, F^{\prime \prime \prime}(t) d t=d v$, then we get

$$
X_{1}=2\left(u v-\int v d u\right)=2\left[t F^{\prime \prime}(t)-F^{\prime}(t)\right]
$$




$$
\begin{aligned}
& X_{2}=\left(t^{2}-1\right) F^{\prime \prime}(t)-2\left[t F^{\prime}(t)-F(t)\right] \\
& \frac{1}{i} X_{3}=\left(t^{2}+1\right) F^{\prime \prime}(t)-2\left[t F^{\prime}(t)-F(t)\right]
\end{aligned}
$$

where $F(t)$ is a differentiable function of $\mathrm{t}$ and $t \in C$.

\section{REFERENCES}

1. Müller, H.R., Sphärische Kinematik,VEB Deutscher Verlag der Wissenschaften, Berlin, 1962.

2. Blaschke, W. und Müller, H.R.: Ebene Kinematik, R. Oldenbourg, München, 1956.

3. Müller, H. R.: Kinematik Dersleri, A.Ü. F. F. Yayınları, Ankara, Turkey, 1963.

4. Hacisalihoğlu, H.H.: Hareket Geometrisi ve Kuaterniyonlar Teorisi, G.Ü. F-E Fak. Y. Mat. No.2, 1983.

5. Yang, An, Tzu : Application of Quaternion Algebra and Dual Numbers to the Analysis of Spatial Mechanizm, Colombia University, Eng. Sc. D. 1963, Ph. D. Dissertation.

6. Willis, A.P.: Vector analysis with an introduction to tensor analysis, New York: Dover Publications, 1958.

7. Y1lmaz, E.: Introduction to Minimal Curves and Surfaces , E.Ü.F.B.Ens.Master Thesis, İzmir, 2002.

8. Hamilton, W.R.: On a new space of imaginary quantities connected with a theory of quaternians, Dublin Proc., Vol-2, no.13, November 1843.

9. Blaschke,W. :Vorlesungen Über Differential Geometrie I. Verlag von Julieus Sipringer in Berlin 1930. 